\title{
A Low-Noise Direct Incremental A/D Converter for FET-Based THz Imaging Detectors
}

\author{
Moustafa Khatib * (D) and Matteo Perenzoni \\ Center for Materials and Microsystems, Integrated Radiation and Image Sensors Group, \\ Fondazione Bruno Kessler, Trento 38122, Italy; perenzoni@fbk.eu \\ * Correspondence: khatib@fbk.eu; Tel.: +39-3891-629956
}

Received: 20 March 2018; Accepted: 5 June 2018; Published: 7 June 2018

check for updates

\begin{abstract}
This paper presents the design, implementation and characterization results of a pixel-level readout chain integrated with a FET-based terahertz $(\mathrm{THz})$ detector for imaging applications. The readout chain is fabricated in a standard 150-nm CMOS technology and contains a cascade of a preamplification and noise reduction stage based on a parametric chopper amplifier and a direct analog-to-digital conversion by means of an incremental $\Sigma \Delta$ converter, performing a lock-in operation with modulated sources. The FET detector is integrated with an on-chip antenna operating in the frequency range of $325-375 \mathrm{GHz}$ and compliant with all process design rules. The cascade of the FET $\mathrm{THz}$ detector and readout chain is evaluated in terms of responsivity and Noise Equivalent Power (NEP) measurements. The measured readout input-referred noise of $1.6 \mu \mathrm{V}_{r m s}$ allows preserving the FET detector sensitivity by achieving a minimum NEP of $376 \mathrm{pW} / \sqrt{\mathrm{Hz}}$ in the optimum bias condition, while directly providing a digital output. The integrated readout chain features $65-\mathrm{dB}$ peak-SNR and $80-\mu \mathrm{W}$ power consumption from a $1.8-\mathrm{V}$ supply. The area of the antenna-coupled FET detector and the readout chain fits a pixel pitch of $455 \mu \mathrm{m}$, which is suitable for pixel array implementation. The proposed $\mathrm{THz}$ pixel has been successfully applied for imaging of concealed objects in a paper envelope under continuous-wave illumination.
\end{abstract}

Keywords: readout circuit; field-effect transistor (FET); terahertz imaging; direct detectors; incremental ADC; flicker noise; lock-in; chopper; parametric amplifier

\section{Introduction}

Recent advancement in $\mathrm{THz}$ technology for developing imaging systems has drawn a great deal of attention [1,2]. Imaging at $\mathrm{THz}$ frequencies provides many interesting and peculiar properties, such as the non-ionizing nature and the low photon energy of the radiation, making it an inherently safe technology, a relatively high resolution (with respect to microwaves) and, lastly, the capability of penetrating several materials such as clothes, plastics and paper. Accordingly, $\mathrm{THz}$ radiation holds great promise for a vast variety of commercial applications ranging from security screening of concealed threats [3] to biomedical diagnosis [4] and from food inspection [5] and quality control [6] to non-destructive material testing [7].

Therefore, the recent research was devoted to the development of efficient detectors that could achieve a room-temperature performance comparable to commercially available detectors and compatible with industrial CMOS technologies. THz direct detectors were prominent, since they have successfully demonstrated adequate detection performance and modest sensitivity without using heterodyne techniques that require a complex experimental setup [8]. Among the good candidates of $\mathrm{THz}$ direct detectors, microbolometers [9], Schottky Barrier Diodes (SBDs) [10] and Field-Effect Transistors (FETs) [11] have been customized to build THz Focal Plane Arrays (FPAs) for imaging applications. 
Typically, microbolometers require specialized process technologies, such as a post-CMOS micromachining, or above-IC wafer processing to make them compatible with CMOS technologies. Moreover, they need to operate in a particular environment (e.g., vacuum packaging) to achieve their typical sensitivity, which dramatically increases the fabrication complexity and cost. On the other hand, CMOS-based THz detectors such as SBDs and FETs are more suitable for cost-effective, video-rate imaging, thanks to CMOS technology that facilitates system-on-chip solutions through a standard fabrication process with high integration capabilities. SBDs were originally used for microwave detection, because of their high sensitivity and their ability to operate at ambient or cryogenic temperatures. However, they still have the issues of non-availability as a standard cell in CMOS and the need to bias them with a current, which directly increases the $1 / f$ noise. In addition, their fabrication process still suffers from significant performance fluctuations when realized in large imaging arrays [12]. Instead, FETs have the advantage that they are not limited by their cut-off frequency as in SBDs due to the plasmonic behavior that takes place inside the transistor's channel enabling the $\mathrm{THz}$ detection.

The major challenges for FET-based THz detectors are related to the low detected signal intensity, which is of the order of a few tens of microvolts with a limited bandwidth, since the frame rate goes from several tens of $\mathrm{Hz}$ to a few $\mathrm{kHz}$ in the case of $\mathrm{THz}$ imaging applications. With this signal characteristic, the low-frequency flicker noise and the DC-offset severely influence the FET detector sensitivity and therefore degrade the dynamic range of the imaging system. However, this can be addressed by amplifying the weak FET signal, while suppressing the flicker noise and the DC-offset and, hence, achieving a high Signal-to-Noise Ratio (SNR) within the detector's signal bandwidth.

A standard technique to reduce the noise level consists of externally interfacing the FET detector with a lock-in amplifier for measuring its weak DC voltage signal. However, this technique is not a feasible approach for simultaneous readout of multi-pixel FET detector arrays with pixels acquiring instantaneously the signal at the $\mathrm{kHz}$-frame rate. Instead, a CMOS-based readout interface can be potentially integrated inside the pixel for performing the signal amplification and filtering.

In the literature, several approaches for the readout interface have been presented, attempting to enhance FET detector sensitivity. In [13], a $3 \times 5$ FPA for imaging at $650 \mathrm{GHz}$ has been presented, with a readout circuit realizing a differential amplifier, which was a source of significant noise and therefore degraded the system performance. Moreover, THz characterization of such an FPA is still dependent on the lock-in technique to obtain raster-scanned $\mathrm{THz}$ images with very slow frame rates. The work in [14] presented an architecture for a FET-based 1-k pixel camera for video imaging. In this architecture, an integration capacitor of $8 \mathrm{pF}$ per pixel is utilized to decrease the integrated noise by reducing the system cut-off frequency, but still not filtering all the noise down to the signal bandwidth. Other work in [15] efficiently addressed the noise filtering by using a demodulation chain based on high-Q Switched-Capacitor (SC) filtering. However, the $1 / f$ noise reduction depends on the possibility of modulating the $\mathrm{THz}$ source at hundreds of $\mathrm{kHz}$, which is not a cost-effective solution for $\mathrm{THz}$ imaging system. A more recent work in [16] presented an FPA for imaging at $860 \mathrm{GHz}$, integrated with a single-readout chain containing a cascade of a chopper instrumentation amplifier and a sigma-delta ADC. Despite the achieved THz performance parameters of the imaging system, the large area of the readout circuit makes it not be compact to be fully integrated inside each pixel for simultaneous readout of multi-pixel imaging arrays. Moreover, the power consumption is expected to be large due to the noise constraints with such a complex readout structure.

In this work, we present the design and complete $\mathrm{THz}$ characterization of a low noise readout chain integrated with a FET THz detector, capable of preserving its minimum NEP at a low modulation frequency $(<1 \mathrm{kHz})$. The proposed design aims, in terms of size and power consumption, to constitute a building block for a future focal-plane array implementation. A preliminary characterization of the proposed structure was previously presented in $[17,18]$. In Section 2 , the design considerations for the FET THz detector are explained. Section 3 describes the architecture and circuit design of the proposed readout chain. Section 4 presents the implementation of the $\mathrm{THz}$ pixel structure. Moreover, 
the $\mathrm{THz}$ characterization and imaging setups along with the experimental results are discussed. Lastly, Section 5 presents the conclusions of the work.

\section{Antenna-Coupled FET Detector}

\subsection{FET-Based THz Detector}

The FET THz detection mechanism has been clarified by plasma wave theory, in which the transistor channel is modeled as an electron gas with a hydrodynamic behavior [19]. Then, the distributed resistive self-mixing theory [20] modeled the transistor channel as a Non-Quasi-Static (NQS) R-C ladder network in a more circuit-focused understanding. In both theories, the FET transistor performs a self-modulation, therefore rectifying the incident $\mathrm{THz}$ radiation induced by the integrated antenna into a DC signal at its drain terminal. A DC gate bias voltage is also provided to control the modulation inside the transistor channel.

The FET detector performance is mainly described by two key indicators, which can be clarified by Figure 1 . First is the detector responsivity $\left(R_{V}, \mathrm{~V} / \mathrm{W}\right)$, which is essentially dependent on the power coupling efficiency from the integrated antenna, as well as the antenna-detector impedance-matching condition. Both are highly related to the effects of parasitic elements between the gate and the drain under certain gate bias voltage. It is observed in [21] that, by designing the FET with minimum dimensions and tuning its gate voltage to reach an optimum bias point, a considerable improvement in the responsivity can be achieved at the proper impedance matching. Figure 2 shows the FET input impedance as a function of the gate bias voltage. The FET impedance has been calculated as described in [22], according to the process model parameters in the chosen CMOS technology at $325 \mathrm{GHz}$. While it is true that $R_{d s}$ decreases with high $V_{g s}$, the real part of the FET impedance increases with $V_{g s}$ according to the given model because it is based on a combination of parallel/series connections and it does not necessarily have the same behavior as $R_{d s}$, as shown in [16].

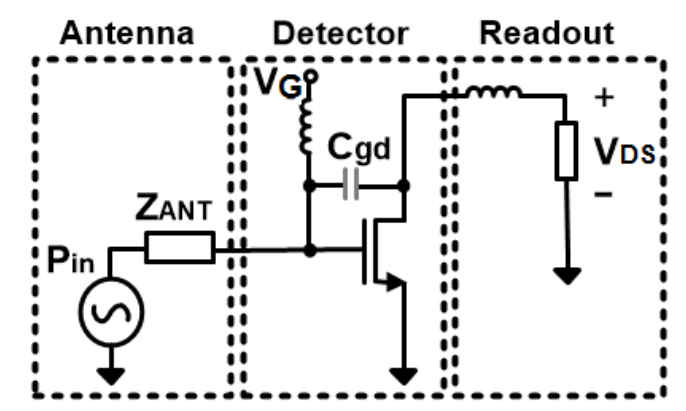

Figure 1. FET-based $\mathrm{THz}$ detector model.

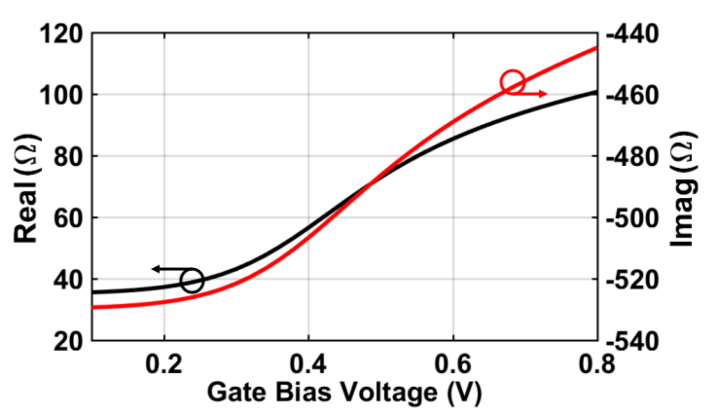

Figure 2. Simulated FET input impedance versus the gate bias voltage at $325 \mathrm{GHz}$.

Next, the Noise Equivalent Power (NEP, W $/ \sqrt{\mathrm{Hz}}$ ), which evaluates the FET detector sensitivity, is defined as the ratio between the FET's output noise voltage spectral density $(\mathrm{V} / \sqrt{\mathrm{Hz}})$ and the 
voltage responsivity $R_{V}$. In a cold-biased configuration, the FET's drain current $I_{D}$ is nearly negligible, and thus, the flicker noise has no relevant influence on the detector sensitivity. Thus, the FET noise contribution is mainly due to the thermal noise voltage of the channel resistance $R_{d s}$ as expressed by:

$$
V_{n}=\sqrt{4 k_{B} T R_{d s} \Delta f}
$$

where $k_{B}$ is the Boltzmann constant, $T$ is the temperature and $\Delta f$ is the detector's noise bandwidth. At optimum gate bias voltage, a minimum NEP can be achieved. Based on the chosen CMOS process, we anticipate that a transistor with minimum feature size $(W / L=0.32 \mu \mathrm{m} / 0.15 \mu \mathrm{m})$ gives a minimum $\mathrm{NEP}$ at $V_{g s}=300 \mathrm{mV}$. Therefore, the channel resistance $R_{d s}=800 \mathrm{k} \Omega$ at this bias point introduces a thermal noise voltage of $3.64 \mu \mathrm{V}_{r m s}$ for a detector noise bandwidth of $1 \mathrm{kHz}$. Therefore, the integrated readout chain must be designed with the input referred noise specification well below this value to maintain the FET detector sensitivity.

\subsection{On-Chip Bow-Tie Antenna Design}

A differential bow-tie antenna has been designed to operate in the frequency range of 325-375 GHz according to the methodology described in [23]. Figure 3 describes the antenna structure with the metal stack of the CMOS technology. The antenna cell dimensions are $455 \times 320 \mu^{2}$. The adopted CMOS process provides six metal layers, with a thick metal option, sandwiched between layers of a dielectric with a relative permittivity $\left(\epsilon_{r}\right)$ of 4.1. The bow-tie is built by the top thick metal layer (MT), while the bottom layer M1 is utilized as a ground plane in order to shield the substrate and avoid the losses caused by the surface waves. The dielectric height from the ground plane to the bow-tie is approximately $7 \mu \mathrm{m}$. The remaining layers (M2-M6) are utilized as square dummy patches to achieve the required metal density in order to meet the process design rules of CMOS technology. Furthermore, the top thick metal layer (MT) is compliant with the foundry rules without any dummy because of antenna arms. The gate and source terminals of the FET detector are directly connected to the differential antenna through stacked metal vias. The antenna performance has been validated through CST Microwave studio EM simulations. In order to transfer the maximum power, the antenna impedance and the FET impedance have to be a complex conjugate pair. The antenna impedance $\left(Z_{A N T}\right)$ versus the signal frequency is plotted in Figure 4a, indicating $Z_{A N T}=(146+497 j) \Omega$ at a frequency of $325 \mathrm{GHz}$. As is visible in Figure $4 \mathrm{~b}$, the radiation efficiency is in the range of $26-33 \%$ from 325 to $375 \mathrm{GHz}$ with a range of directivity of 4.5-5.1 dBi. In fact, the obtained efficiency is low due to the thin dielectric layer between the bow-tie and the ground plane, in addition to the conductor and dielectric losses at this frequency range. However, we do not expect sidelobes due to the surface waves thanks to the reflector.

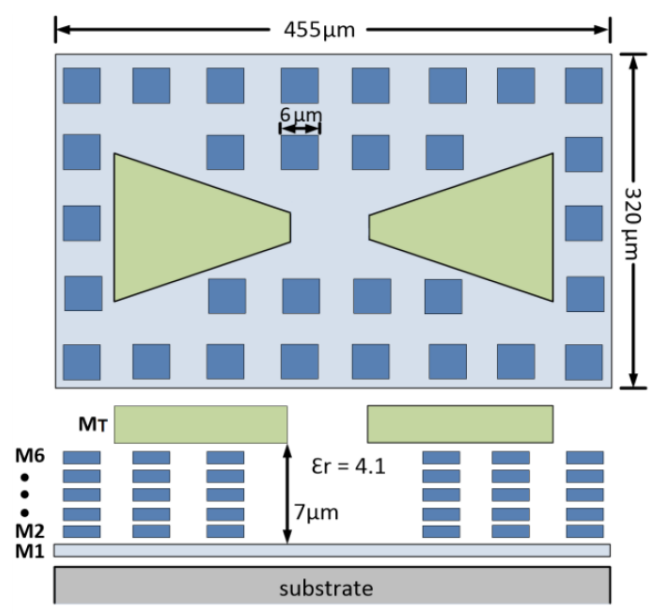

Figure 3. Design of the differential bow-tie antenna in the adopted 150-nm CMOS technology. 


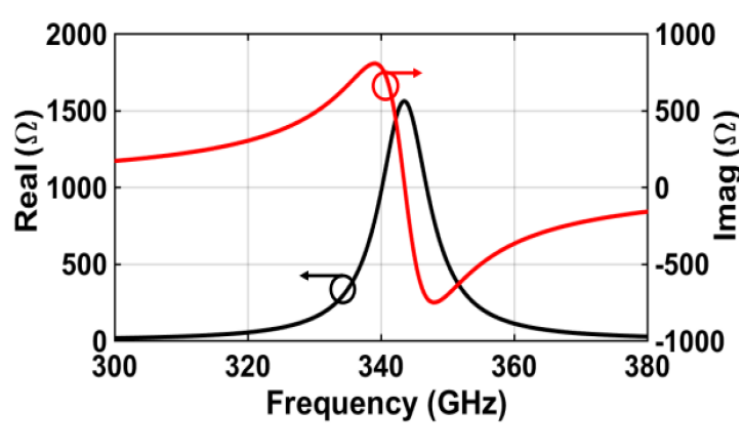

(a)

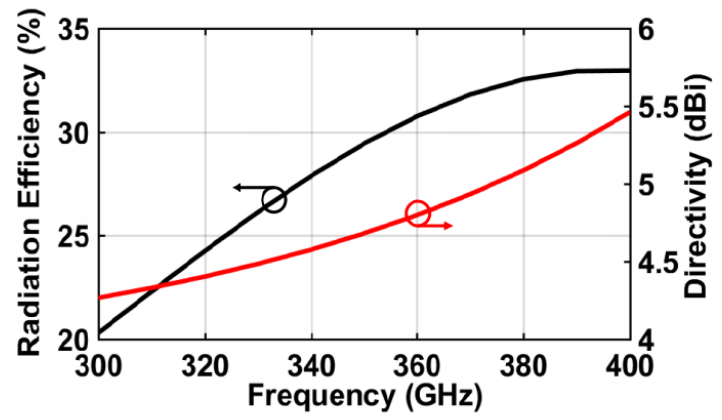

(b)

Figure 4. Simulation results of the bow-tie antenna: antenna impedance (a); antenna radiation efficiency and directivity (b).

\section{THz Readout Chain Design}

Figure 5 illustrates the block diagram of the FET THz detector circuit integrated with the readout chain. The FET THz detector is realized by two NMOS transistors in a gate-driven configuration: One of them is connected to the integrated antenna, acting as an active FET THz detector. The second NMOS is acting as a blind detector, providing a reference voltage for offset compensation of the differential input pair, and thus, only the voltage difference between the two detectors is amplified. The two detectors are provided with the same gate bias voltage. The integrated readout chain is composed of a cascade of a preamplification and noise reduction stage based on a parametric chopper amplifier and a direct analog-to-digital conversion by means of an incremental $\Sigma \Delta$ converter followed by a decimation stage that provides a 12-bit digital output as a measure of the intensity of the rectified FET signal.

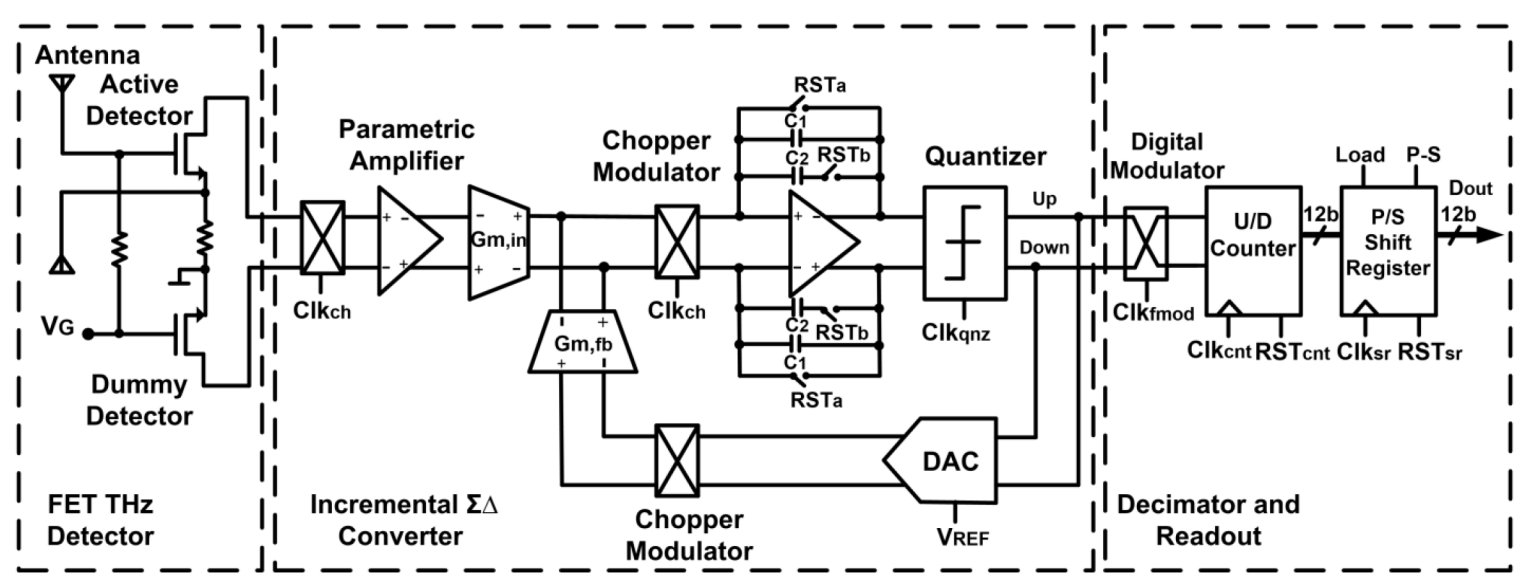

Figure 5. Block diagram of the proposed $\mathrm{THz}$ detector and readout structure.

\subsection{Parametric Chopper Amplification}

The operation principle of the parametric amplifier is schematically clarified in Figure 6. The parametric amplifier operates in a discrete-time mode, containing two sampling switches followed by two MOS varactors [24]. The amplification process has the advantage of being noise-free with low power consumption [25]. The obtained gain is basically given as the ratio of the small-signal gate capacitances of the MOS varactors through alternating the signals $C l k_{s}$ and $\mathrm{Clk}_{\text {boost }}$ in two non-overlapped clock phases. The parametric amplifier is enclosed by the chopper modulators that operate at a frequency of $f_{c h}$, which is half of the operating frequency of the parametric amplifier. 
Therefore, they eliminate the $1 / f$ noise from the signal path simultaneously along with reducing the thermal noise during the sampling and the boost phases of the parametric amplifier.

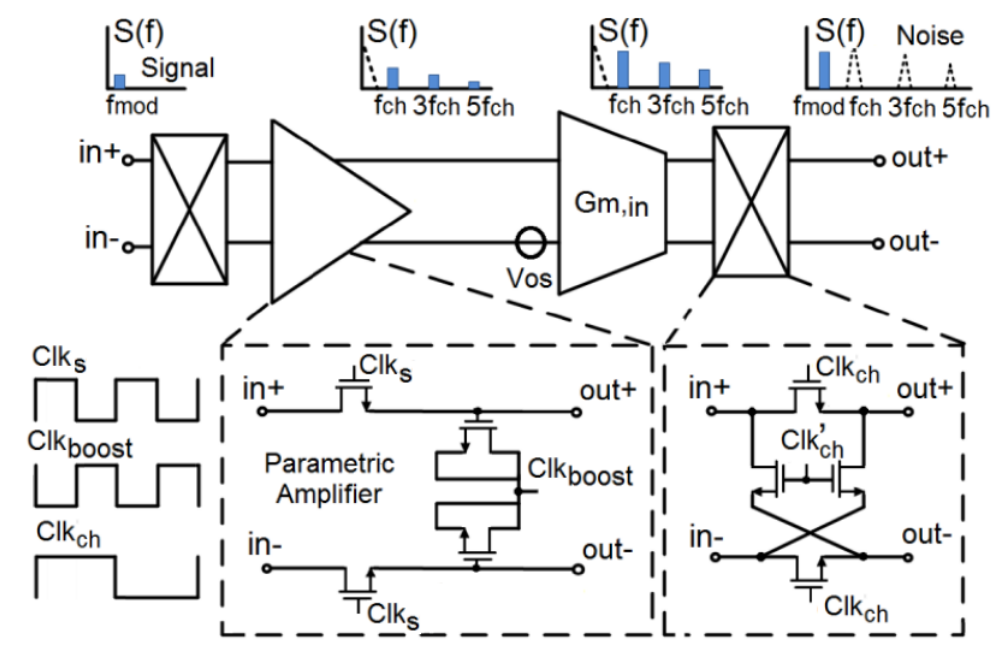

Figure 6. $1 / f$ noise and offset cancellation by using the parametric chopper amplifier.

The value of the passive gain is approximately $2-3$, reducing the overall input referred thermal noise contribution of the readout chain. Figure 7 shows the simulations of the gain and noise of the chopper parametric amplifier, performed by Parametric Steady-State, AC and Noise (PSS/PAC and PNOISE) analyses. The simulations show a negligible noise contribution of $9 \mathrm{nV} / \sqrt{\mathrm{Hz}}$ dominated by only thermal noise with a passive voltage gain of 2.3 at a chopping frequency of $100 \mathrm{kHz}$. Three choppers are placed around the parametric amplifier and the transconductors in the feed-forward and feedback paths to reduce $1 / f$ noise and the DC-offset. Only the feed-forward path is considered in Figure 6 for clarification. The first chopper at the input modulates the FET signal, which is located at the source modulation frequency $f_{\text {mod }}$, to the odd harmonics of the chopping frequency $f_{c h}$. Then, the modulated signal together with $1 / f$ noise and the DC-offset are amplified by a certain gain provided by the loop filter ( $\mathrm{G}_{m}$ stages with the Miller integrator) of the incremental ADC. Afterwards, the last chopper at the transconductor output demodulates the signal back to $f_{\text {mod }}$ and shifts the $1 / f$ noise and the offset to the odd harmonics of chopping frequency, such that they will be filtered out by the loop filter. All the switches in the chopper modulators are implemented with a complementary transmission gate with minimum transistor dimensions, to reduce the chopper spikes caused by the charge injection during the switching between the two clock phases.

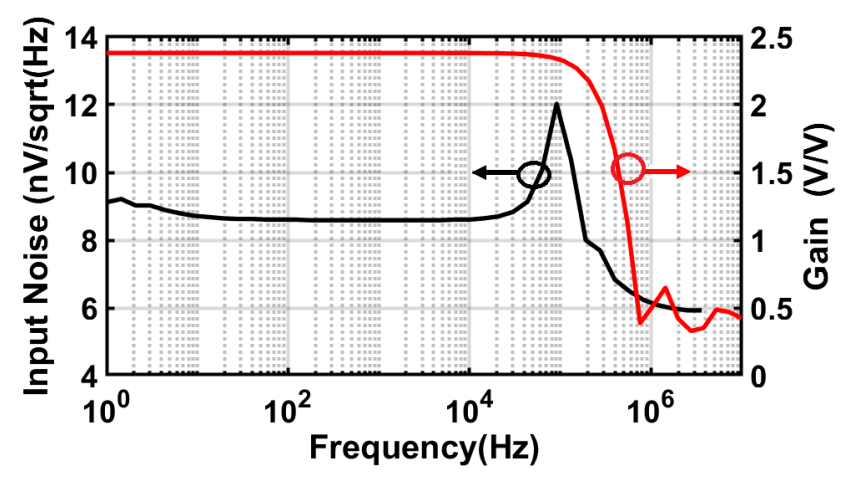

Figure 7. Gain and noise simulation results of the parametric amplifier at a chopping frequency of $100 \mathrm{kHz}$. 


\subsection{Continuous-Time Incremental Conversion}

Incremental sigma-delta $(\Sigma \Delta)$ Analog-to-Digital Converters (ADCs) benefit from the oversampling and noise shaping techniques with relaxed matching requirements similar to traditional $\Sigma \Delta$ ADCs [26]. The incremental $\Sigma \Delta$ operation offers the advantage of simultaneously integrating the rectified FET signal and averaging the detector noise, providing a one-to-one mapping between the FET signal and the digital output at each conversion cycle [27].

In this design, a first order modulator structure is realized, since it offers a medium resolution (i.e., more than 10 bits) that fulfills the requirements of $\mathrm{THz}$ imaging applications, without increasing the complexity in both the loop filter and the decimator [28]. A Continuous-Time (CT) loop filter based on a $G_{m}-C$ structure has been implemented, since it relaxes the settling and bandwidth requirements when compared to the switched-capacitor counterpart, and hence, it achieves lower power consumption [29]. The operating principle of the readout chain is described in the conceptual timing diagram of Figure 8. The CT loop filter and the decimator are reset at the beginning of each conversion cycle $\left(T_{\text {conv }}\right)$. Then, the slowly varying FET voltage signal $\left(V_{F E T}\right)$ is continuously integrated and sampled for an integration period of $T_{i n t}=2^{n} T_{q n z}$, where $n$ is the converter resolution and $T_{q n z}$ is the sampling period. In each period of $T_{q n z}$, the integrator output voltage is compared to the quantizer threshold and a decision is made. Next, depending on the quantizer decision, the voltage DAC feeds back either a $+V_{R E F}$ or a $-V_{R E F}$ signal to the input of the feedback transconductor; accordingly, the up-down digital counter, which is realized as a decimator, will either increment or decrement its value. At the end of $T_{\text {conv }}$, the integrator output voltage can be described by:

$$
V_{\text {int }}=\frac{G_{m_{\text {in }}}}{C_{\text {int }}} T_{q n z}\left[2^{n} V_{F E T}-\frac{G_{m_{f b}}}{G_{m_{\text {in }}}}\left(N_{u p}-N_{\text {down }}\right) V_{R E F}\right]
$$

where $C_{i n t}=C_{1}+C_{2}, G_{m_{i n}}$ and $G_{m_{f b}}$ are the integrating capacitor and effective transconductance values in the feed-forward and feedback paths, respectively. $N_{u p}$ and $N_{\text {down }}$ are the number of subtractions and additions of $V_{R E F}$ respectively. The Least Significant Bit (LSB) resolution is thus defined by $\mathrm{V}_{R E F}$ scaled by both of the transconductance values in the feed-forward and feedback paths, as well as the number of clock cycles per one conversion as given by:

$$
V_{L S B}=\left(\frac{G_{m_{i n}}}{G_{m_{f b}}}\right)\left(\frac{V_{R E F}}{2^{n}}\right)
$$

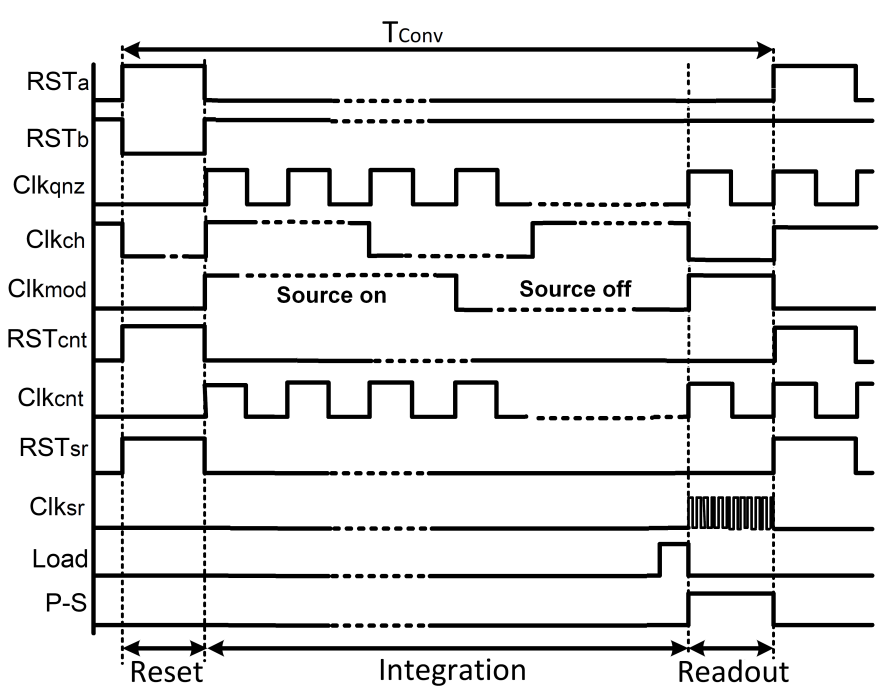

Figure 8. Timing diagram of the $\mathrm{THz}$ readout chain. 
Finally, the digital counter value is extracted by a parallel-to-serial shift register and a new conversion cycle begins with cleared counter. The digital modulator is realized between the quantizer and the counter and operates at the same frequency of the source $f_{\text {mod }}$, thus, enabling the measurement of the pixel output in both conditions (source on/source off) so as to calculate the difference between them, similar to the lock-in technique. Figure 9 shows the schematic of the implemented decimator.

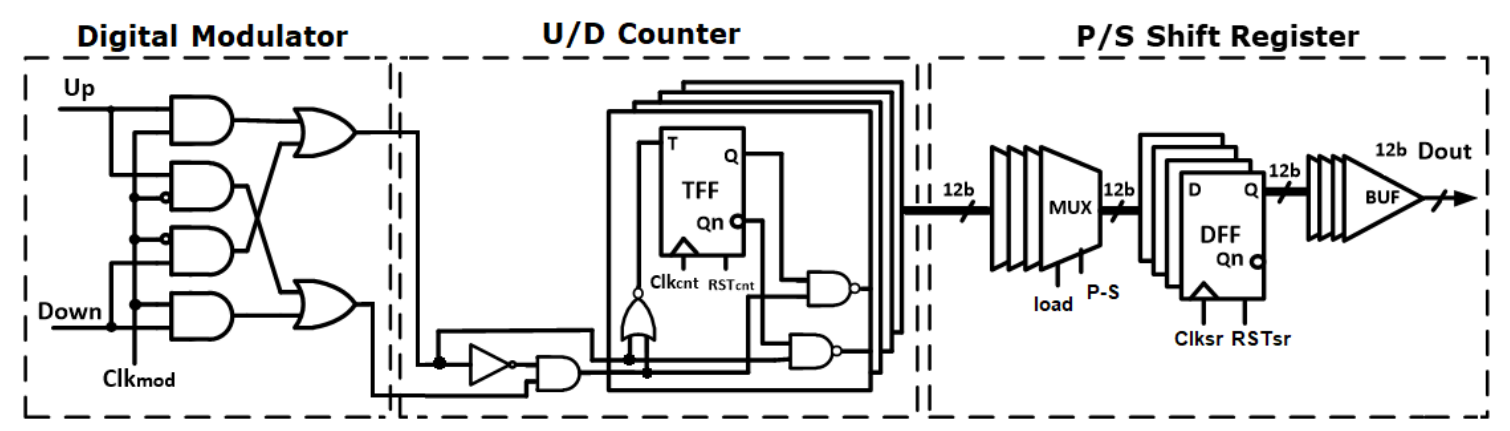

Figure 9. Schematic of the implemented decimator.

The transconductors ( $G_{m}$ stages), depicted in Figure 10a, are designed using a pseudo-differential source degeneration topology with resistors acting as transconducting elements [30]. In principle, the follower transistors (M1-M2) transfer the input voltage to the resistor, consequently improving the linearity in the V-I conversion. Then, the current mirrors (M3-M6) transfer the currents to the outputs. Therefore, the effective $G_{m}$ value will be approximately equal to $1 / R$. PMOS transistors operating in weak inversion were used for the input differential pair due to their lower $1 / f$ noise contribution and to satisfy the input common-mode specification for the FET voltage signals. All the other transistors are sized to have a large length and operated in strong inversion such that the noise should be only dominated by the input pair.

The output currents from the $G_{m}$ stages are added/subtracted and then injected into a Miller integrator [31], so that the effect of finite output impedance at the transconductors' outputs is reduced. The amplifier employed in the Miller integrator is composed of a current buffer common-gate input followed by a common-source stage as visible in Figure 10b. The bias current in the $G_{m}$ stages and the Miller integrator, which sets the noise floor of the incremental ADC, is $4 \mu \mathrm{A}$, resulting in a total current dissipated in the incremental loop filter of $24 \mu \mathrm{A}$.

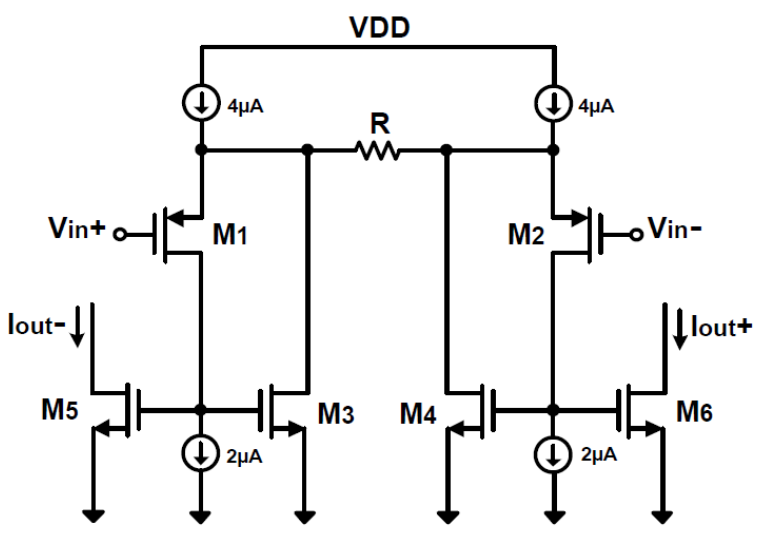

(a)

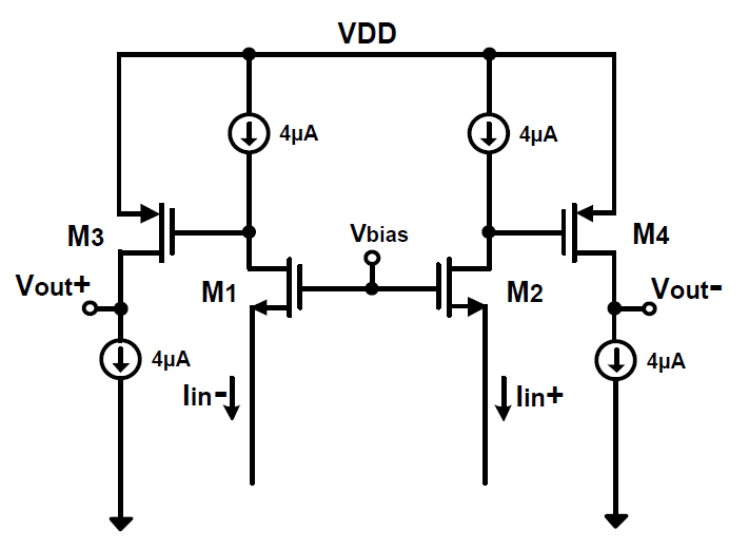

(b)

Figure 10. Schematic of the pseudo-differential $G_{m}-C$ loop filter: (a) transconductor; and (b) the amplifier used in the Miller integrator. 
Figure 11 shows the schematic of the implemented single-bit quantizer. It is based on the structure presented in [32], containing a pre-amplifier stage to avoid the kick-back noise, followed by a positive feedback latched comparator. The implemented comparator is designed with minimum transistor size, except for the bias transistor $M_{\text {tail }}$, which was scaled with respect to a current mirror-based bias circuit. Transient simulations were performed to scale the coefficients of the CT loop transfer function and guarantee that the integrator outputs are within a proper bound (i.e., $\pm V_{R E F}$ ). The integrating capacitors are sized to be $800 \mathrm{fF}$, while the $G_{m}$ values in the feed-forward and feedback paths are scaled to be $130.33 \mu \mathrm{S}$ and $3.33 \mu \mathrm{S}$, equivalent to degeneration resistors of $7.5 \mathrm{k} \Omega$ and $300 \mathrm{k} \Omega$, respectively. The loop filter can provide an amplification for the integrated FET signal by a voltage gain that can be evaluated as,

$$
\text { Gain }=\left(\frac{2 G_{m_{i n}}}{C_{i n t}}\right) T_{C o n v}
$$

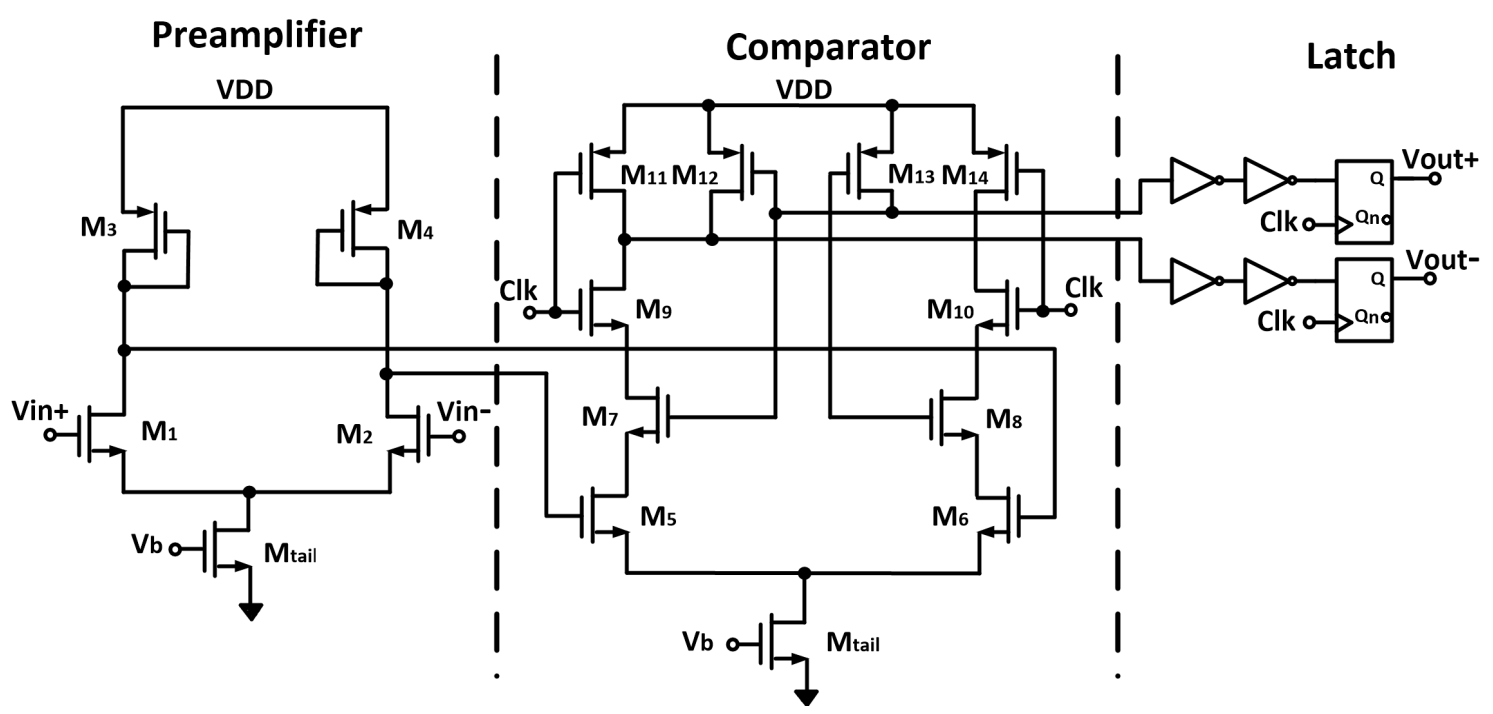

Figure 11. Schematic of the implemented single-bit quantizer.

According to [33], the chopping frequency is chosen to be: (i) several times greater than the $1 / f$ noise corner (which is around $10 \mathrm{kHz}$ from the noise simulation), to effectively eliminate the flicker noise; and (ii) below the bandwidth of the loop filter in order to suppress the chopper spikes. The integrating capacitors are utilized as Metal-Insulator-Metal (MIM) capacitors, while high-resistivity polysilicon is used for realizing the degeneration resistors.

\section{THz Characterization and Measurements}

The design has been fabricated in a 150-nm standard CMOS technology. A micrograph of the pixel structure is shown in Figure 12 with an inset demonstrating the detailed layout of different blocks of the readout chain realized in a total area of $90 \times 300 \mu \mathrm{m}^{2}$. Two identical readout circuits are implemented: one of them is integrated with the antenna-coupled FET THz detector, and the other is for the purpose of performing an electrical test for the readout circuit operation. 


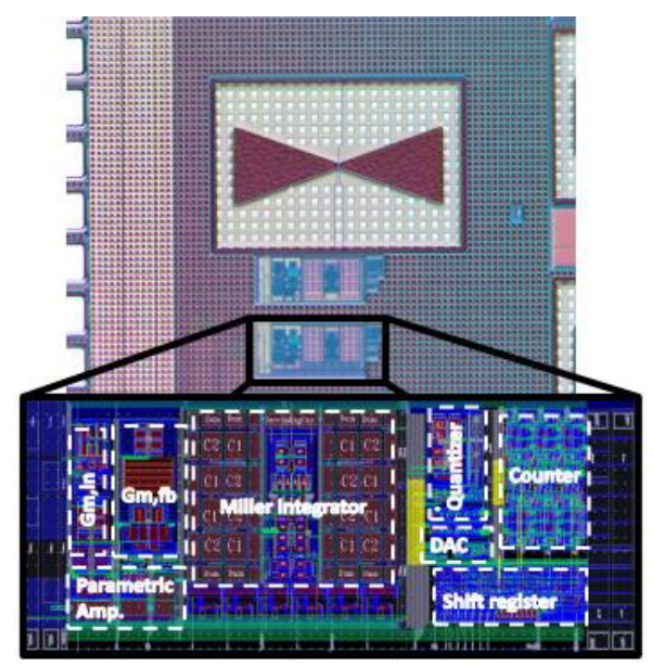

Figure 12. Micrograph of the fabricated $\mathrm{THz}$ pixel structure.

\subsection{Electrical Measurements of the Readout Chain}

The measurement of the input-referred noise power is performed in three different configurations of the $\mathrm{THz}$ readout chain as presented in Figure 13a. The noise measurements were acquired at the CT loop filter output, while the differential input pins are shorted to ground. Then, it is divided by the total closed-loop gain in order to be referred to the input of the readout chain. The noise measurements are obtained at a chopping frequency of $100 \mathrm{kHz}$ to effectively eliminate the $1 / f$ noise and the DC-offset in the signal bandwidth.

It can be seen that the flicker noise is dominant at low frequency when the choppers are not active. In the case of bypassing the parametric amplifier and only using the conventional chopper technique, the flicker noise is effectively reduced, and only the thermal noise remains in the signal bandwidth. The input noise spectrum density averages around $-139 \mathrm{~dB} / \mathrm{Hz}$ at lower frequencies. However, when a passive gain of $2-3$ is provided by the parametric amplifier at the beginning of the readout chain, the thermal noise is further reduced by more than $6 \mathrm{~dB}$. The measured input noise remains around $-146 \mathrm{~dB} / \mathrm{Hz}$, which corresponds to a total integrated noise of $1.6 \mu \mathrm{V}_{r m s}$ over a $1-\mathrm{kHz}$ bandwidth. By comparing this measured value to the intrinsic noise originated by the FET detector, which is around $3.64 \mu \mathrm{V}_{r m s}$, the readout can efficiently preserve the minimum NEP of the FET detector that is only limited by its thermal noise voltage of the channel resistance. Figure $13 \mathrm{~b}$ demonstrates the simulation of the total input noise power of the readout chain: when no noise reduction technique is applied and when the chopper parametric amplifier is operating at a chopping frequency of $100 \mathrm{kHz}$, showing a good agreement with the measurement results.

To validate the performance of the incremental sigma-delta converter, a small amplitude $500-\mathrm{Hz}$ sine wave has been injected into the readout chain. A Fast Fourier Transform (FFT) has been performed on the quantizer output by using a broadband oscilloscope. Figure 14a shows the measured graph of the output signal Power Spectral Density (PSD) showing a first order quantization noise shaping with an oversampling rate of 500, which is in a good agreement with the simulated one. Moreover, the output noise power spectral density is presented in Figure 14b. The measured SNR is 65 dB, which is equivalent to be 10.6 effective number of bits $(E N O B)$, evaluated as [26]:

$$
E N O B=\log _{2}\left(\frac{2 V_{i n, \max }}{V_{L S B}}\right)
$$

where $V_{i n, \max }$ is the maximum input voltage. The sampling rate of the incremental converter is set as a tradeoff between the bandwidth of the CT loop filter and the desired resolution, which depends on the 
number of clock cycles. Since the FET-detected signals have a maximum bandwidth of $1 \mathrm{kHz}$, a high sampling rate is not necessary. Therefore, the quantizer is designed to operate with a sampling frequency of $1 \mathrm{MHz}$ to achieve an Oversampling Ratio (OSR) of 500 for the incremental conversion operation.

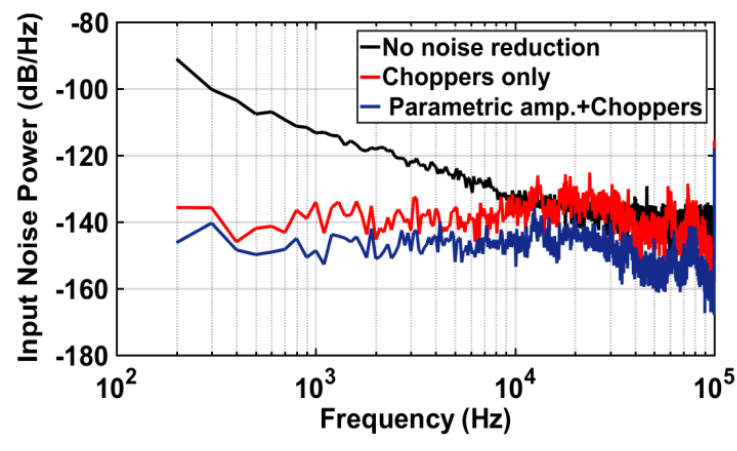

(a)

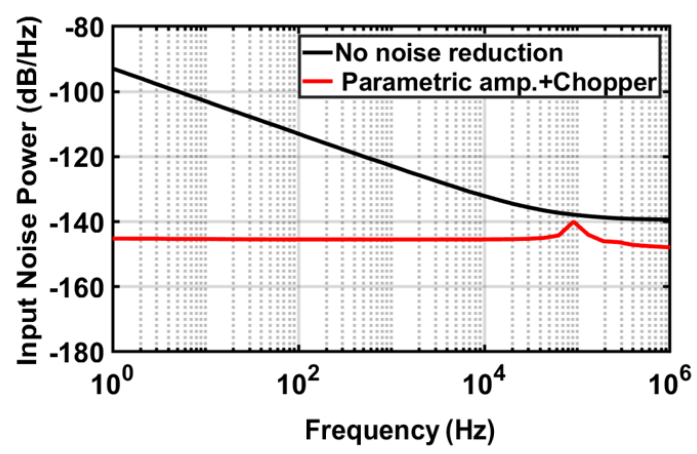

(b)

Figure 13. (a) Measured input noise power: without noise reduction (black), with the conventional chopper technique (red) and with the proposed parametric chopper amplification (blue), chopping $f=100 \mathrm{kHz}$; and (b) simulation of the input noise power of the readout chain: without noise reduction (black) and with the chopper parametric amplifier (red).

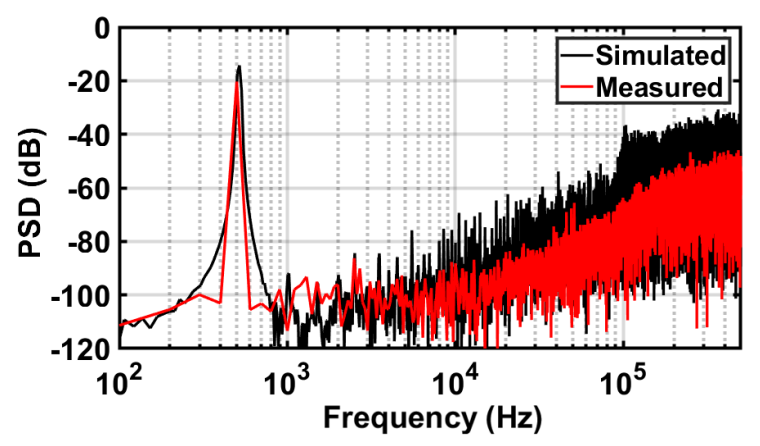

(a)

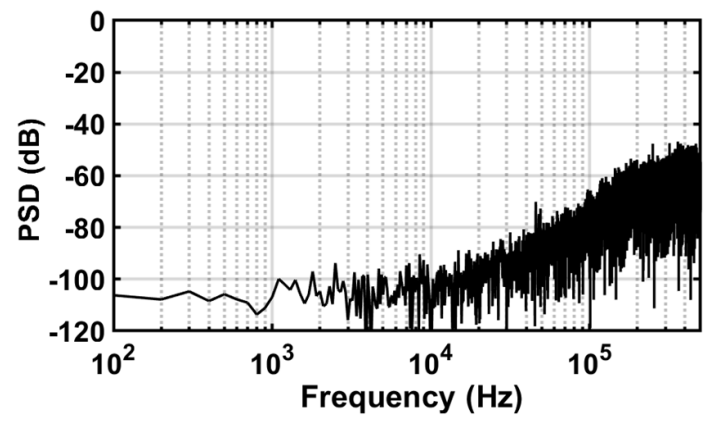

(b)

Figure 14. Simulated and measured output signal PSD of the incremental sigma-delta converter tested with an input sinusoidal tone at $500 \mathrm{~Hz}$ and sampling rate $1 \mathrm{MHz}$ (a); and noise PSD measured with shorted input to ground (b).

\section{2. $\mathrm{THz}$ Experimental Setup}

The experimental setup utilized to characterize the FET detector and the readout chain is illustrated in Figure 15. A Continuous-Wave (CW) frequency synthesizer with a multiplier chain was used as the THz source to generate a signal with frequencies between 265 and $375 \mathrm{GHz}$. Afterward, the generated signal was transmitted into free space through a horn antenna. A Zeonex lens, with a focal length of $5 \mathrm{~cm}$, was used to focus the THz beam on the chip. The amplitude of $\mathrm{THz}$ signal was electrically modulated at different frequencies by using a function generator.

A control and acquisition board was used to generate the necessary control waveforms and to obtain the measured responses from the readout chain. In addition, a User-Controlled Attenuation (UCA) switch performed background noise subtraction measurements, hence removing any coupling or interference in the setup. According to the measurement methodology described in [34], the impinging input power received by the FET detector was measured by obtaining the impinging 
power density of a pyroelectric device as a reference detector with known characteristics. Then, it was multiplied by the antenna effective area $A_{e f f}$, as evaluated by:

$$
A_{e f f}=\frac{D \lambda^{2}}{4 \pi}
$$

where $D$ is the antenna directivity and $\lambda$ is the wavelength of the THz signal. The impinging power delivered to the FET detector was estimated as:

$$
P_{\text {FET }}=\left(\frac{V_{\text {Pyro }}}{R_{\text {pyro }}}\right)\left(\frac{A_{\text {eff }}}{A_{\text {Pyro }}}\right)
$$

where $A_{\text {Pyro }}, V_{\text {Pyro }}$ and $R_{\text {pyro }}$ are the pyroelectric sensitive area, the measured pyroelectric voltage response and its responsivity, respectively.

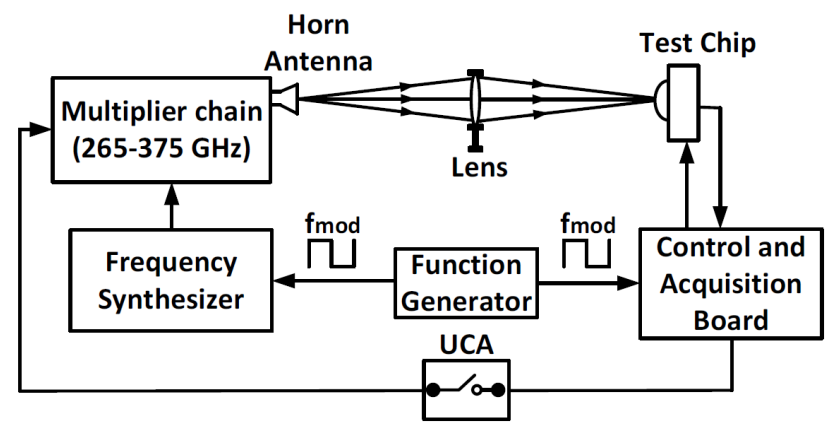

Figure 15. Block diagram of the $\mathrm{THz}$ characterization setup.

\subsection{Antenna-Coupled FET Detector Measurements}

Initially, the performance of a standalone antenna-coupled FET THz detector was evaluated through a lock-in amplifier with sensitivity and time constant of $500 \mu \mathrm{V}$ and $200 \mathrm{~ms}$, respectively. The FET voltage responsivity was evaluated as the measured FET voltage response $V_{F E T}$ normalized by the impinging power $P_{F E T}$. By sweeping the gate bias from the sub-threshold to a strong inversion region, as is visible in Figure 16a, the FET detector achieved a peak responsivity of $318 \mathrm{~V} / \mathrm{W}$ at a gate bias voltage of about $0.3 \mathrm{~V}$; while it decreased towards both: (i) the strong inversion region, since the $R_{d s}$ was reduced, and thus, the output voltage drop across it decreases, as well, and (ii) the sub-threshold, due to the lowering of the cutoff frequency of the FET-lock-in interface well- below the source modulation frequency $[35,36]$. The FET detector output noise voltage spectrum density was measured around $115 \mathrm{nV} / \sqrt{\mathrm{Hz}}$, which was equivalent to the thermal noise contribution originated from the measured FET channel resistance $R_{d s}=800 \mathrm{k} \Omega$ at a gate bias voltage of $0.3 \mathrm{~V}$, as shown in Figure 17. Then, NEP can be estimated by dividing the measured thermal noise voltage by the FET responsivity, that is,

$$
N E P=\frac{\sqrt{4 k_{B} T R_{d s}}}{R_{V}}
$$

Correspondingly, a minimum NEP of $281 \mathrm{pW} / \sqrt{\mathrm{Hz}}$ was obtained at the same gate bias point of $0.3 \mathrm{~V}$. Figure $16 \mathrm{~b}$ shows the frequency sweep analysis for the FET responsivity with an inset illustrating the THz beam spot sensed by the FET detector. The beam spot was acquired on an X-Y transverse plane to the optical axis by scanning the test chip in 0.4-mm steps using a stepper motor. It is also possible to notice that the FET detector responsivity is above $200 \mathrm{~V} / \mathrm{W}$ from $355-375 \mathrm{GHz}$ with a peak value of $318 \mathrm{~V} / \mathrm{W}$ near $365 \mathrm{GHz}$. 


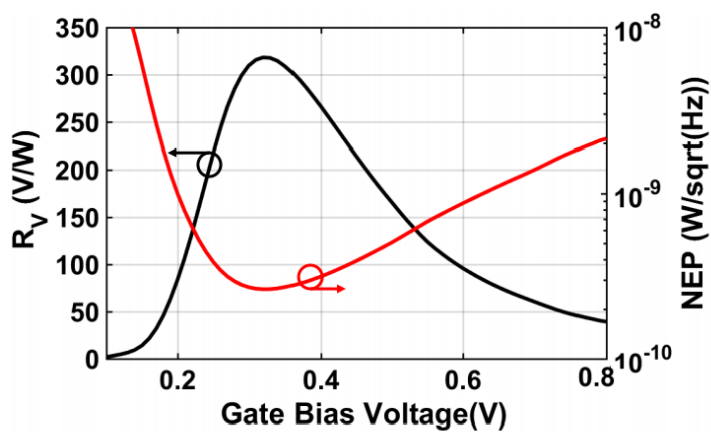

(a)

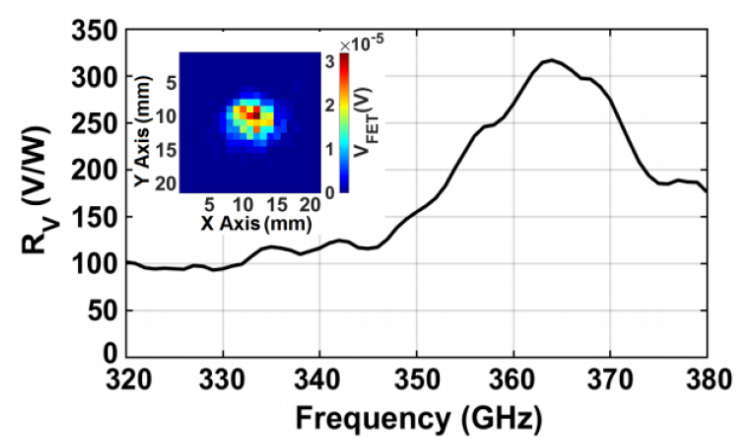

(b)

Figure 16. Measured FET voltage responsivity and Noise Equivalent Power (NEP) versus gate bias voltage (a); and measured FET voltage responsivity versus signal frequency (b).

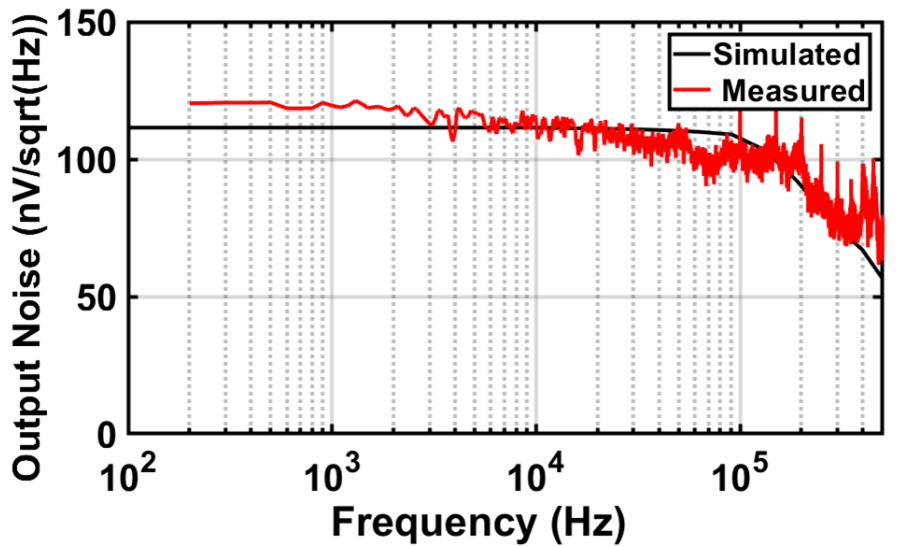

Figure 17. Simulated and measured FET detector noise voltage spectral density versus frequency.

\subsection{Readout Responsivity and NEP Measurements}

Measurements of responsivity and NEP for the FET THz detector were eventually acquired through the implemented readout chain, in place of the external lock-in amplifier. The measurements were performed by modulating the $\mathrm{THz}$ source and the digital modulator (see Figure 5) at three different frequencies within the range of the FET detector bandwidth of $1 \mathrm{kHz}$.

Thanks to the digital modulator, the recorded digital response was the net value of the difference between the two states of the modulating signal waveform (while the THz signal was present and while there was no $\mathrm{THz}$ signal), such that it could perform the lock-in function. The voltage response of the FET detector was recorded as 12-bit digital code through the shift register output per each conversion cycle. The integration time of the readout chain was correlated to the modulation frequency of the FET signals $(<1 \mathrm{kHz})$, which was typically in the range of $1-10 \mathrm{~ms}$. The measured responsivity as a function of gate bias voltage is presented in Figure 18a at different modulation frequencies, showing a peak response near a $0.3-\mathrm{V}$ gate bias voltage.

Since the readout directly converts the FET response to a digital output without any representative voltage except the detector's, we need to rewrite the responsivity, which then would not be comparable to $R_{V}$ of course, but the NEP still gave a metric for comparison because it was input-referred. The responsivity was expressed as a digital number per unit of impinging power (DN/W) instead of $(\mathrm{V} / \mathrm{W})$, as given by:

$$
R_{\text {dig }}=\frac{D N}{P_{F E T}}
$$

Similarly, Figure $18 \mathrm{~b}$ shows the responsivity as a function of signal frequency, exhibiting peak values near 365 GHz. NEP curves in Figure 19 were measured by calculating the standard deviation of 
several acquired digital outputs $\delta_{D N}$, i.e., the RMS of the output code of each conversion cycle, divided by the measured responsivity acquired at a signal frequency of $365 \mathrm{GHz}$. Then, it was divided by the square root of the FET bandwidth (e.g., $f_{\text {mod }}$ ), as given by:

$$
N E P=\frac{\delta_{D N}}{R_{\text {dig }} \sqrt{f_{\text {mod }}}}
$$

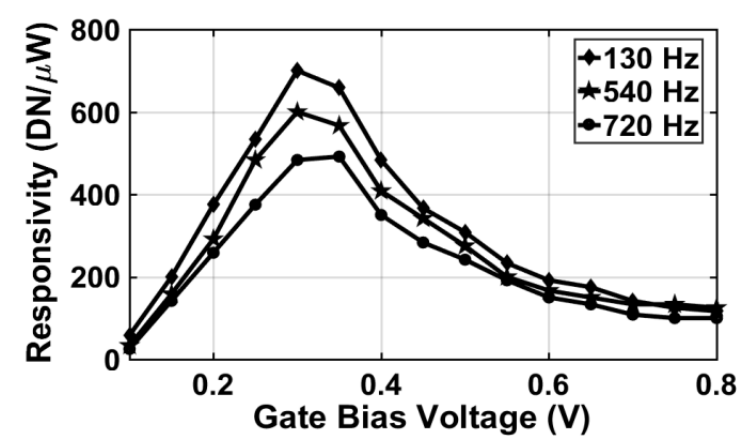

(a)

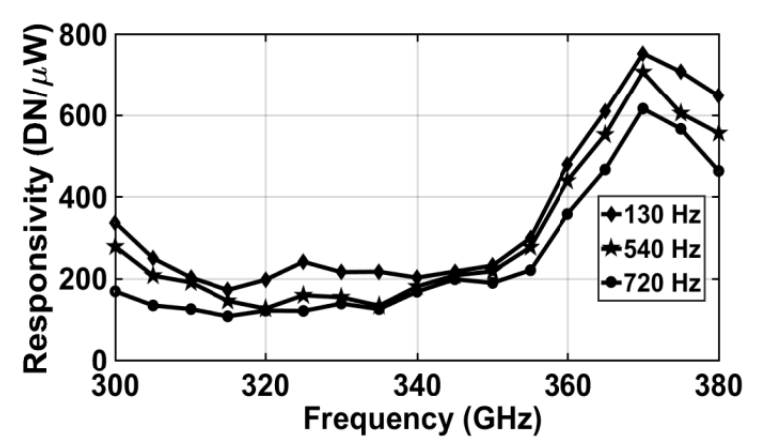

(b)

Figure 18. Readout responsivity as a function of FET gate bias voltage (a) and signal frequency (b).

The minimum NEP value of $376 \mathrm{pW} / \sqrt{\mathrm{Hz}}$ was obtained at a gate bias voltage of $0.3 \mathrm{~V}$ and a modulation frequency of $130 \mathrm{~Hz}$. The measured NEP was only due to the thermal noise contribution of the FET detector without being influenced by the readout noise, since the measured input noise power was significantly below the FET thermal noise. However, due to the fact that the dummy FET detector contributes to the thermal noise voltage by its channel resistance, the obtained NEP by the readout chain was approximately $\sqrt{2}$-times higher than the measured NEP of a single FET detector. The NEP of both active and dummy FETs is visible in the dashed line in Figure 19, exhibiting a good match with the readout measurements. It can be seen that the total NEP (FETs + readout) was generating less noise than FETs only at a lower gate voltage due to the fact that the responsivity of the detector alone was lower in that range due to the RC time constant (e.g., the connection to the instrumentation), which was not present with the readout chain.

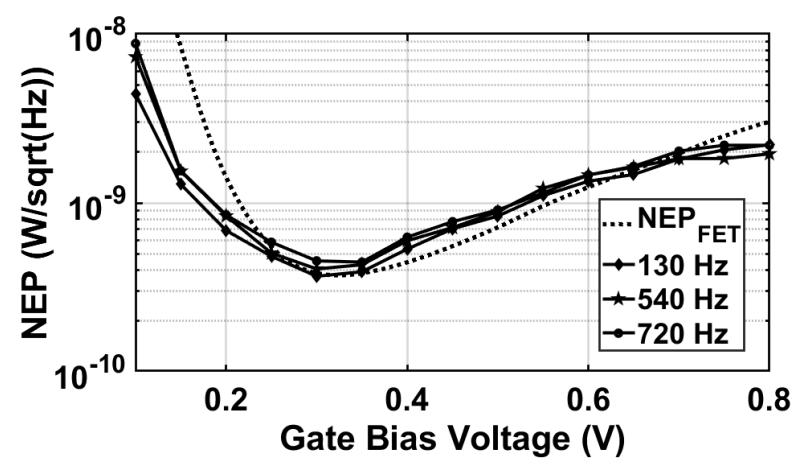

Figure 19. NEP as a function of FET gate bias voltage (measured at $365 \mathrm{GHz}$ ).

\section{5. $\mathrm{THz}$ Imaging}

The optical experimental setup used for performing $\mathrm{THz}$ imaging acquisition is presented in Figure 20. The image object was vertically positioned in the focal point between two Zeonex lenses, such that the THz beam was focused on the object by the first lens and collimated by the second lens and then refocused on the test chip. Since our sensor contains only a single pixel, mechanical scanning was required to obtain a wide field of view. A stepper motor stage was used to scan the objects in 
the vertical and horizontal directions by a step size of $0.4 \mathrm{~mm}$. Metallic and plastic objects concealed inside a paper envelope were mechanically scanned and captured in transmission mode at $365 \mathrm{GHz}$ and a modulation frequency of $130 \mathrm{~Hz}$. As shown in Figure 21, the scanned images clearly resolve the structural details of the objects such as screws, a SIM card, a nut and a metallic ring. The scanned area is $20 \times 20 \mathrm{~mm}^{2}$ with a total resolution of $50 \times 50$ pixels.

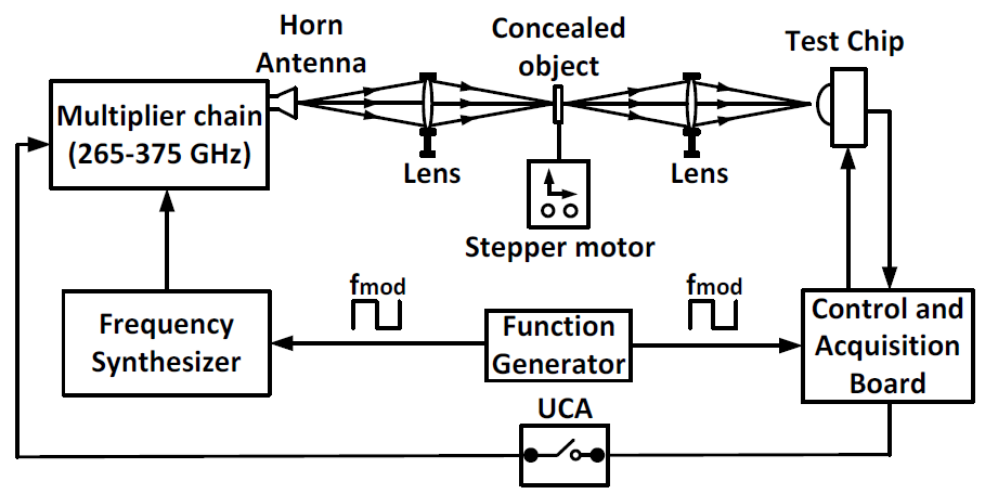

Figure 20. Block diagram of the $\mathrm{THz}$ imaging setup.
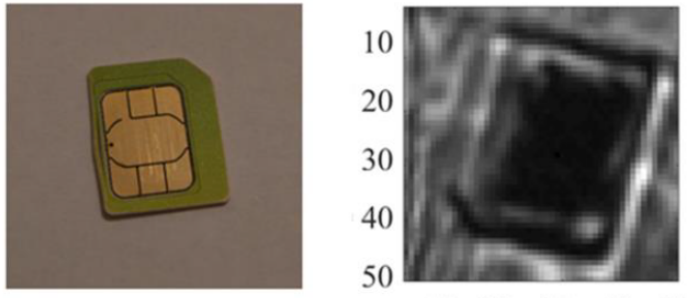

$\begin{array}{lllll}10 & 20 & 30 & 40 & 50\end{array}$

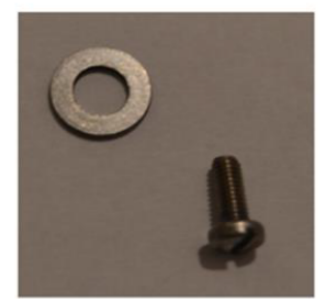

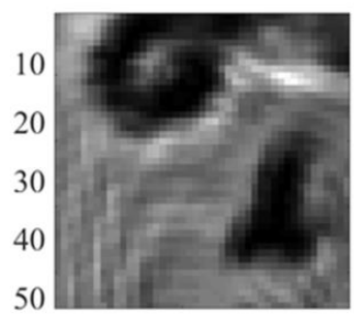

$\begin{array}{lllll}10 & 20 & 30 & 40 & 50\end{array}$
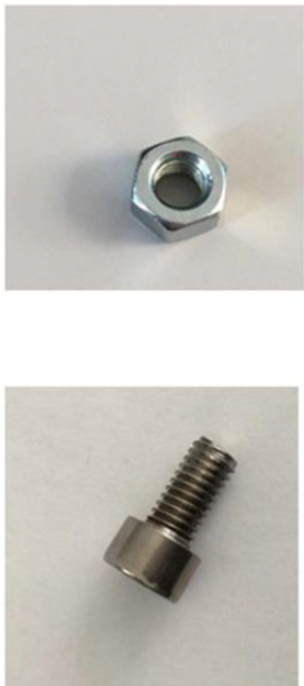
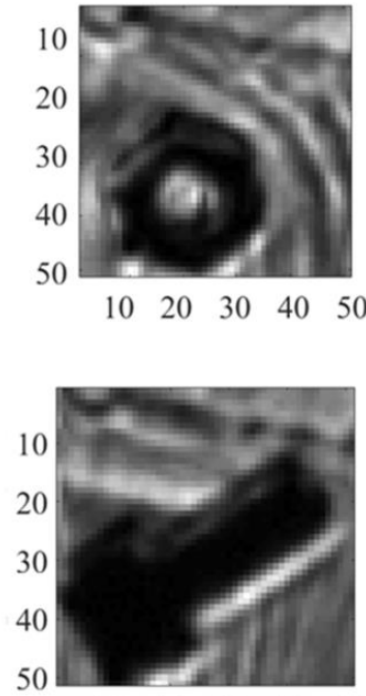

$\begin{array}{lllll}10 & 20 & 30 & 40 & 50\end{array}$

Figure 21. THz images of different metallic/plastic objects hidden inside a paper envelope acquired at $365 \mathrm{GHz}$ (source modulation $f=130 \mathrm{~Hz}$ ) along with the photographs of the objects.

The total acquisition time of each image was around $3.5 \mathrm{~h}$, due to the limited speed of the stepper motors, which was essentially dominated by the actuator's speed, while the effective acquisition time amounted to just several seconds. The background interference pattern appearing in the images was produced by the standing waves inside the paper envelope. In comparison with the conventional lock-in technique, typically the lock-in amplifier required an integration time in the range of 200-500 ms in order to achieve similar signal quality, while the proposed readout chain acquires the data of each pixel during an integration period in the range of 1-10 ms, depending on the applied modulation frequency. This is regardless of the time consumed by the stepper motors to move between different pixels. The overall performance of the presented $\mathrm{THz}$ pixel structure is summarized in Table 1 and compared to the recently-reported state of the art. The proposed THz pixel features: (1) a first order incremental ADC that is compact in terms of area and power and can be fully integrated inside the $\mathrm{THz}$ pixel, providing simultaneous integration and readout; (2) suitable for pixels also with smaller 
antennas (e.g., $800 \mathrm{GHz}$ ); (3) it does not require sources with a high modulation frequency, so that the cost of the THz imaging system could be significantly reduced; (4) a direct conversion to the digital domain, which means robust and easy signal management. (5) Low input-referred noise.

Table 1. Performance comparison with the recently reported state of the art.

\begin{tabular}{cccccc}
\hline & This Work & T-TST'17 [16] & Sensor'16 [15] & JSSC'12 [14] & JSSC'09 [13] \\
\hline Process & $0.15 \mu \mathrm{m}$ & $0.18 \mu \mathrm{m}$ & $0.13 \mu \mathrm{m}$ & $65 \mathrm{~nm}$ & $0.25 \mu \mathrm{m}$ \\
Input-Referred Noise & $1.6 \mu \mathrm{V}_{r m s}$ & $2.03 \mu \mathrm{V}_{r m s}$ & $0.2 \mu \mathrm{V}_{r m s}$ & $2.45 \mu \mathrm{V}_{r m s}$ & - \\
Power Consumption & $80 \mu \mathrm{W}$ & - & $320 \mu \mathrm{W}$ & $2.5 \mu \mathrm{W}$ & $5.5 \mathrm{~mW}$ \\
Source Frequency & $325-375 \mathrm{GHz}$ & $860 \mathrm{GHz}$ & $270 \mathrm{GHz}$ & $856 \mathrm{GHz}$ & $650 \mathrm{GHz}$ \\
Modulation Frequency & $10 \mathrm{~Hz}-1 \mathrm{kHz}$ & $177 \mathrm{~Hz}$ & $156 \mathrm{kHz}$ & $5 \mathrm{kHz}$ & $30 \mathrm{kHz}$ \\
On-chip Antenna & Bow-tie antenna & Patch antenna & Bow-tie antenna & Ring antenna & Patch antenna \\
Pixel Size & $455 \times 455 \mu \mathrm{m}^{2}$ & $1330 \times 440 \mu \mathrm{m}^{2}$ & $240 \times 240 \mu \mathrm{m}^{2}$ & $80 \times 80 \mu \mathrm{m}^{2}$ & $200 \times 150 \mu \mathrm{m}^{2}$ \\
Maximum Responsivity & $783 \mathrm{DN} / \mu \mathrm{W}$ & $3.3 \mathrm{kV} / \mathrm{W}$ & $300 \mathrm{kV} / \mathrm{W}$ & $140 \mathrm{kV} / \mathrm{W}$ & $80 \mathrm{kV} / \mathrm{W}$ \\
Minimum NEP & $376 \mathrm{pW} / \sqrt{\mathrm{Hz}}$ & $106 \mathrm{pW} / \sqrt{\mathrm{Hz}}$ & $533 \mathrm{pW}$ & $12 \mathrm{nW}$ & $300 \mathrm{pW} / \sqrt{\mathrm{Hz}}$ \\
& $@ 130 \mathrm{~Hz}$ & $@ 177 \mathrm{~Hz}$ & $@ 156 \mathrm{kHz}$ & $@ 500 \mathrm{kHz}$ & $@ 30 \mathrm{kHz}$ \\
\hline
\end{tabular}

\section{Conclusions}

In this paper, a noise efficient readout chain integrated with an antenna-coupled FET THz detector has been developed in 150-nm CMOS technology. The electrical measurements and $\mathrm{THz}$ characterization demonstrate the performance of the readout chain, which effectively performs the function of integrated lock-in amplification with a direct digital output. Owing to the in-pixel parametric chopper technique and the anti-aliasing filtering of the incremental conversion loop, the flicker noise and the DC-offset are effectively eliminated without modulating the $\mathrm{THz}$ source at high frequencies. The integrated readout noise of $1.6 \mu \mathrm{V}_{r m s}$ over a $1-\mathrm{kHz}$ bandwidth resulted in a peak-SNR of $65 \mathrm{~dB}$, sufficient for obtaining a good signal quality for THz imaging applications. The detection behavior of the FET detector with the readout chain has shown a good sensitivity with a minimum NEP value of $376 \mathrm{pW} / \sqrt{\mathrm{Hz}}$ at $365 \mathrm{GHz}$, while providing a direct digital responsivity. Moreover, the acquired $\mathrm{THz}$ images through the readout chain with its compact pixel size of $455 \times 455 \mu \mathrm{m}^{2}$ and the low power consumption of $80 \mu \mathrm{W}$ have demonstrated good performance as detectors in $\mathrm{THz}$ imaging.

Author Contributions: M.K. contributed to the readout circuit design, carried out the theoretical analysis and all the experimental characterizations and wrote this article. M.P. contributed to the antenna design and provided supervision and guidance for this work. All the authors have read and approved the final manuscript.

Conflicts of Interest: The authors declare no conflict of interest.

\section{References}

1. Jansen, C.; Wietzke, S.; Peters, O.; Scheller, M.; Vieweg, N.; Salhi, M.; Krumbholz, N.; Jördens, C.; Hochrein, T.; Koch, M. Terahertz imaging: applications and perspectives. Appl. Opt. 2010, 10, E48-E57. [CrossRef] [PubMed]

2. Siege, P.H. Terahertz technology. IEEE Trans. Microw. Theory Technol. 2002, 50, 910-928. [CrossRef]

3. Federici, J.F.; Schulkin, B.; Huang, F.; Gary, D.; Barat, R.; Oliveira, F.; Zimdars, D. THz imaging and sensing for security applications-explosives, weapons and drugs. Semicond. Sci. Technol. 2011, 20, S266-S280. [CrossRef]

4. Ajito, K.; Ueno, Y. THz chemical imaging for biological applications. IEEE Trans. Terahertz Sci. Technol. 2011, 1, 293-300. [CrossRef]

5. Gowen, A.A.; O'Sullivan, C.; O’Donnell, C.P. Terahertz time domain spectroscopy and imaging: emerging techniques for food process monitoring and quality control. Trends Food Sci. Technol. 2012, 25, 40-46. [CrossRef]

6. Rutz, F.; Koch, M.; Khare, S.; Moneke, M.; Richter, H.; Ewert, U. Terahertz quality control of polymeric products. Int. J. Infrared Millim. Waves 2007, 27, 547-556. [CrossRef]

7. Burford, N.M.; El-Shenawee, M.O.; O'Neal, C.B.; Olejniczak, K.J. Terahertz imaging for nondestructive evaluation of packaged power electronic devices. Int. J. Emerg. Technol. Adv. Eng. 2014, 4, 395-401. 
8. Friederich, F.; Spiegel, W.; Bauer, M.; Meng, F.; Thomson, M.; Boppel, S.; Lisauskas, A.; Hils, B.; Krozer, V.; Keil, A.; et al. THz active imaging systems with real-time capabilities. IEEE Trans. Terahertz Sci. Technol. 2011, 1, 183-200. [CrossRef]

9. Oden, J.; Meilhan, J.; Lalanne-Dera, J.; Roux, J.-F.; Garet, F.; Coutaz, J.-L.; Simoens, F. Imaging of broadband terahertz beams using an array of antenna-coupled microbolometers operating at room temperature. Opt. Express 2013, 21, 4817-4825. [CrossRef] [PubMed]

10. Han, R.; Zhang, Y.; Kim, Y.; Kim, D.Y.; Shichijo, H.; Afshari, E.; Kenneth, K.O. Active terahertz imaging using Schottky diodes in CMOS: Array and 860-GHz pixel. IEEE J. Solid State Circuits 2013, 48, 2296-2308. [CrossRef]

11. Lisauskas, A.; Bauer, M.; Boppel, S.; Mundt, M.; Khamaisi, B.; Socher, E.; Venckevičius, R.; Minkevičius, L.; Kašalynas, I.; Seliuta, D.; et al. Exploration of terahertz imaging with silicon MOSFETs. Int. J. Infrared Millim. Terahertz Waves 2014, 35, 63-80. [CrossRef]

12. Han, R.; Zhang, Y.; Coquillat, D.; Videlier, H.; Knap, W.; Brown, E.; Kenneth, K.O. 280-GHz Schottky diode detector in 130-nm digital CMOS. Proc. IEEE Custom Integr. Circuits 2010, 46, 1968-1976.

13. Öjefors, E.; Pfeiffer, U.R.; Lisauskas, A.; Roskos, H.G. A $0.65 \mathrm{THz}$ focal-plane array in a quarter-micron CMOS process technology. IEEE J. Solid State Circuits 2009, 44, 1968-1976. [CrossRef]

14. Al Hadi, R.; Sherry, H.; Grzyb, J.; Zhao, Y.; Forster, W.; Keller, H.M.; Cathelin, A.; Kaiser, A.; Pfeiffer, U.R. A 1 k-Pixel video camera for 0.7-1.1 terahertz Imaging applications in 65-nm CMOS. IEEE J. Solid State Circuits 2012, 47, 2999-3012. [CrossRef]

15. Boukhayma, A.; Dupret, A.; Rostaing, J.P.; Enz, C. A low-noise CMOS THz imager based on source modulation and an in-pixel high-Q passive switched-capacitor N-path filter. Sensors 2016, 16, 325. [CrossRef] [PubMed]

16. Liu, Z.; Liu, L.; Yang, J.; Wu, N.A. CMOS Fully Integrated 860-GHz Terahertz Sensor. IEEE Trans. Terahertz Sci. Technol. 2017, 7, 455-465. [CrossRef]

17. Khatib, M.; Perenzoni, M.; Stoppa, D. A noise-efficient, in-pixel readout for FET-based THz detectors with direct incremental A/D conversion. In Proceedings of the 43rd IEEE European Solid State Circuits Conference, Leuven, Belgium, 11-14 September 2017; pp. 23-26.

18. Khatib, M.; Perenzoni, M.; Stoppa, D. A CMOS 0.15- $\mu \mathrm{m}$ in-pixel noise reduction technique for readout of antenna-coupled FET-based THz detectors. In Proceedings of the 41st International Conference on Infrared, Millimeter, and Terahertz waves (IRMMW-THz), Copenhagen, Denmark, 25-30 September 2016; pp. 1-2.

19. Tauk, R.; Teppe, F.; Boubanga, S.; Coquillat, D.; Knap, W. Plasma wave detection of terahertz radiation by silicon field effects transistors: responsivity and noise equivalent power. Appl. Phys. Lett. 2006, 89, 253511. [CrossRef]

20. Lisauskas, A.; Pfeiffer, U.; Öjefors, E.; Bolivar, P.H.; Glaab, D.; Roskos, H.G. Rational design of high-responsivity detectors of terahertz radiation based on distributed self-mixing in silicon field-effect transistors. J. Appl. Phys. 2009, 105, 114511. [CrossRef]

21. Grzyb, J.; Sherry, H.; Cathelin, A.; Kaiser, A.; Pfeiffer, U.R. On the co-design between on-chip antennas and THz MOSFET direct detectors in CMOS technology. In Proceedings of the 2012 37th International Conference on Infrared, Millimeter, and Terahertz Waves (IRMMW-THz), Wollongong, NSW, Australia, 23-28 September 2012; pp. 1-3.

22. Boppel, S.; Lisauskas, A.; Mundt, M.; Seliuta, D.; Minkevicius, L.; Kasalynas, I.; Valusis, G.; Mittendorff, M.; Winnerl, S.; Krozer, V.; et al. CMOS integrated antenna-coupled field-effect-transistors for the detection of radiation from 0.2 to $4.3 \mathrm{THz}$. IEEE Trans. Microw. Theory Technol. 2012, 60, 3834-3843. [CrossRef]

23. Perenzoni, M.; Cavallo, D. Design of an efficient $900 \mathrm{GHz}$ antenna in standard CMOS technology for imaging arrays. In Proceedings of the 2015 9th European Conference on Antennas and Propagation (EuCAP), Lisbon, Portugal, 13-17 April 2015; pp. 1-2.

24. Ranganathan, S.; Tsividis, Y. A MOS capacitor-based discrete-time parametric amplifier with $1.2 \mathrm{~V}$ output swing and $3 \mathrm{nW}$ power dissipation. In Proceedings of the 2003 IEEE International Solid-State Circuits Conference, San Francisco, CA, USA, 13 February 2003; pp. 406-502.

25. Tsividis, Y.; Suyama, K. Strange ways to use the MOSFET. In Proceedings of the 1997 IEEE International Symposium on Circuits and Systems, Hong Kong, 12 June 1997; pp. 449-452.

26. Tao, S.; Rusu, A. A power-efficient continuous-time incremental sigma-delta ADC for neural recording systems. IEEE Trans. Circuits Syst. I Regul. Pap. 2015, 62, 1489-1498. [CrossRef] 
27. Markus, J.; Silva, J.; Temes, G.C. Theory and applications of incremental $\Delta \Sigma$ converters. IEEE Trans. Circuits Syst. I Regul. Pap. 2004, 51, 678-690. [CrossRef]

28. Liang, J.; Johns, D.A. A frequency-scalable 15-bit incremental ADC for low power sensor applications. In Proceedings of the 2010 IEEE International Symposium on Circuits and Systems (ISCAS), Paris, France, 30 May-2 June 2010; pp. 2418-2421.

29. Khatib, M.; Perenzoni, M. Pixel-level continuous-time incremental sigma-delta A/D converter for $\mathrm{THz}$ sensors. In Proceedings of the Optical Sensing and Detection IV, Brussels, Belgium, 3-7 April 2016.

30. Kwan, T.; Martin, K.W. An adaptive analog continuous-time CMOS biquadratic filter. IEEE J. Solid State Circuits 1991, 26, 859-867. [CrossRef]

31. Willingham, S.D.; Martin, K.W.; Ganesan, A. A BiCMOS low-distortion $8 \mathrm{MHz}$ low-pass filter. IEEE J. Solid State Circuits 1993, 28, 1234-1245. [CrossRef]

32. Allen, P.E.; Holberg, D.R. CMOS Analog Circuit Design, 2nd ed.; Oxford University Press: New York, NY, USA, 2002.

33. Enz, C.; Vittoz, E.A.; Krummenacher, F. A CMOS chopper amplifier. IEEE J. Solid State Circuits 1987, 22, 335-342. [CrossRef]

34. Ali, M.; Perenzoni, M.; Stoppa, D. A methodology to measure input power and effective area for characterization of direct THz detectors. IEEE Trans. Instrum. Meas. 2016, 65, 1225-1231. [CrossRef]

35. Sakowicz, M.; Lifshits, M.B.; Klimenko, O.A.; Schuster, F.; Coquillat, D.; Teppe, F.; Knap, W. Terahertz responsivity of field effect transistors versus their static channel conductivity and loading effects. J. Appl. Phys. 2011, 110, 054512. [CrossRef]

36. Di Gaspare, A.; Casini, R.; Foglietti, V.; Giliberti, V.; Giovine, E.; Ortolani, M. Terahertz current oscillations in a gated two-dimensional electron gas with antenna integrated at the channel ends. Appl. Phys. Lett. 2012, 100, 203504. [CrossRef]

(C) 2018 by the authors. Licensee MDPI, Basel, Switzerland. This article is an open access article distributed under the terms and conditions of the Creative Commons Attribution (CC BY) license (http:/ / creativecommons.org/licenses/by/4.0/). 